\section{Digital dental photography. Part 4: choosing a camera}

\section{Ahmad'}
IN BRIEF
- The most convenient, versatile, easy to use camera for dental applications is the digital single lens reflex (DSLR) camera.
- A high quality lens is the key factor for high resolution images.
- The number of pixels is not an indication of the image quality, but only the size of a digital image.
- Many photographic accessories and dental armamentarium expedite a photographic session.

With so many cameras and systems on the market, making a choice of the right one for your practice needs is a daunting task. As described in Part 1 of this series, a digital single reflex (DSLR) camera is an ideal choice for dental use in enabling the taking of portraits, close-up or macro images of the dentition and study casts. However, for the sake of completion, some other cameras systems that are used in dentistry are also discussed.

The number of cameras on the market is daunting and their specifications mindboggling. The requirements of a camera for dentistry are two-fold: capable of taking portraits, as well as close-up or macro images of the dentition and study casts (Figs 1-3). These dual requirements limit the types of cameras meeting these criteria. Furthermore, a camera system should be flexible, allowing adaptation for changing technology, and versatile for creative photography (Fig. 4). Therefore to simplify the technical jungle, we only need to consider a digital single reflex (DSLR) camera, as

FUNDAMENTALS OF DIGITAL
DENTAL PHOTOGRAPHY
1. Digital dental photography: an overview
2. Purposes and uses
3. Principles of digital photography
4. Choosing a camera and accessories
5. Lighting
6. Camera settings
7. Extra-oral set-ups
8. Intra-oral set-ups
9. Post-image capture processing
10. Printing, publishing and presentations

${ }^{1}$ General Dental Practitioner, The Ridgeway Dental Surgery, 173 The Ridgeway, North Harrow, Middlesex, HA2 7DF

Correspondence to: Irfan Ahmad

Email: iahmadbds@aol.com www.IrfanAhmadTRDS.co.uk

\section{Refereed Paper}

Accepted 15 November 2008

DOI: $10.1038 /$ sj.bdj.2009.

${ }^{\circledR}$ British Dental Journal 2009; 206: 575-581
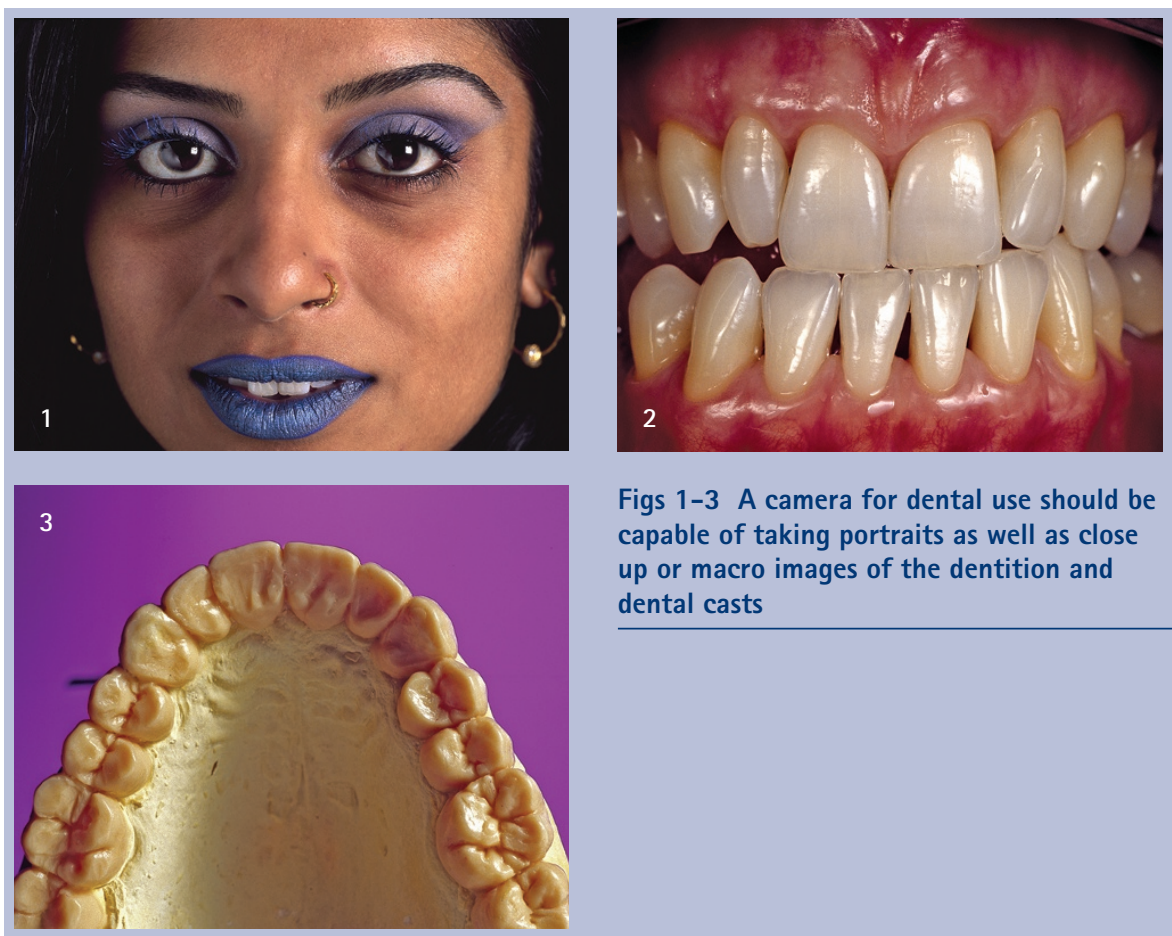

Figs 1-3 A camera for dental use should be capable of taking portraits as well as close up or macro images of the dentition and dental casts

discussed in Part 1, which is an ideal choice for dental use. However, for the sake of completion, a few other cameras systems are discussed that are used in dentistry.

\section{CAMERAS FOR DENTAL PHOTOGRAPHY}

The first is an intra-oral or fibre optic camera. This is an excellent tool for a cursory tour of the oral cavity, showing patients gingival inflammation, decay and defective restorations. While its quality is adequate for displaying on a monitor, it is insufficient for permanent documentation or for archiving.

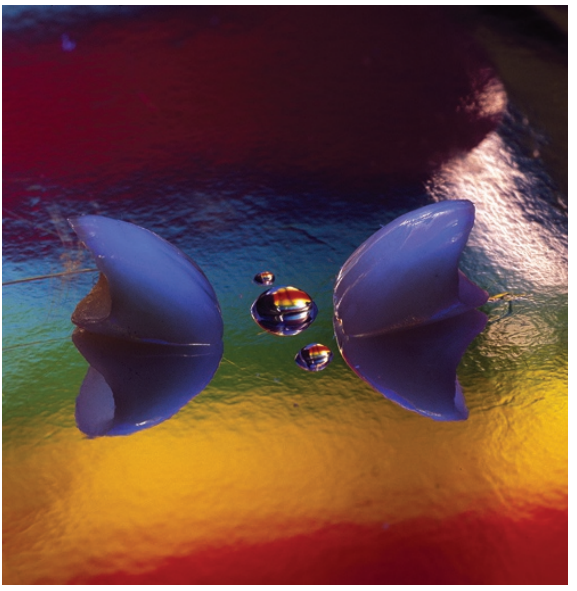

Fig. 4 A versatile camera system allows flexibility for creative images 


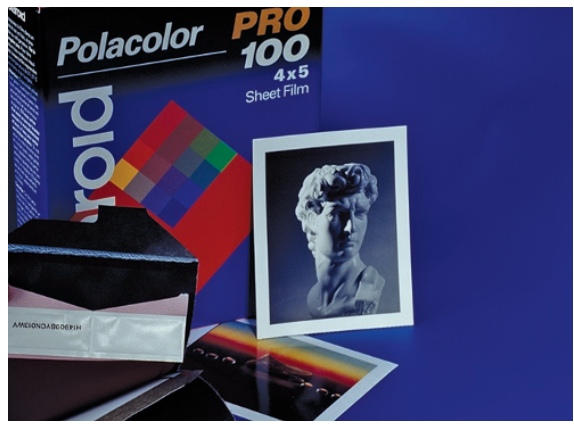

Fig. 5 Before digital photography, Polaroid instant films were the only method of previewing an image before making the final exposure

The second is the Polaroid Marco 5 SLR camera, relatively inexpensive, with automatic exposure, auto-focusing and built-in flashes. Various pre-set magnifications are offered ranging from full face to a few teeth. The 'trademark' of Polaroid is selfdeveloping prints, usually in a few minutes after taking the picture. The images are low quality, unable to be electronically archived, as only one print is produced for each exposure. The film is expensive because each print contains the developing chemicals. Before the advent of digital photography, Polaroid prints were the only method of instantly viewing a picture, but with the arrival of digital technology these cameras have become obsolete, having merely a novelty factor (Fig. 5).

The next tailored camera for dental pictures is the Kodak P712 with an EasyShare docking port printer. This DSLR is adapted from the Kodak budget range of digital cameras, modified specifically for dental use. Lenses, focusing, framing, exposure and flash are all pre-set and automatic. Unlike a Polaroid, images can be stored on a computer and/or immediately printed with the accompanying printer (Fig. 6). The major drawback with this 'shoot and go' camera is the fixed focal length lens, which limits flexibility and versatility. Furthermore, for the same price, a semiprofessional DSLR can be purchased, offering greater possibilities and functionality.

Other cameras are the rangefinder varieties, for example compact 'point and shoot' that are unsuitable for dentistry because they suffer from parallax (Fig. 7). Parallax is defined as the difference between the image seen in a viewfinder and that recorded by a sensor. With macro photography, as the lens moves closer to the subject the variance increases, which means that certain

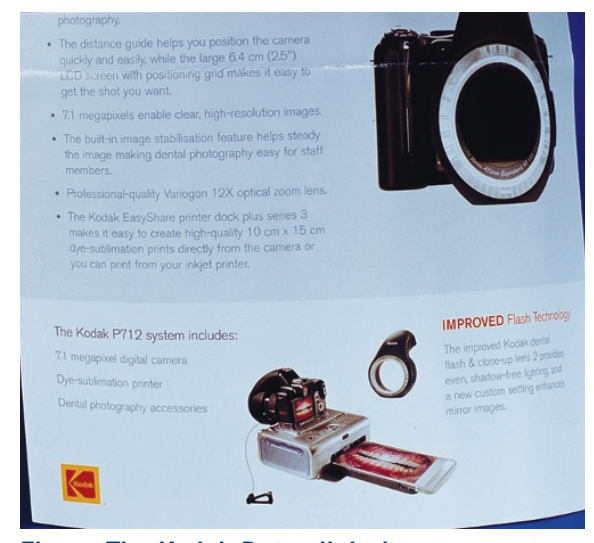

Fig. 6 The Kodak P712 digital camera system

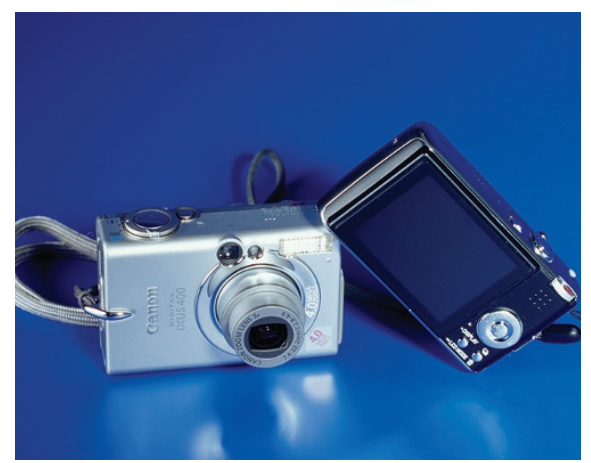

Fig. 7 Compact cameras are unsuitable for dental use due to the disadvantageous parallax phenomenon

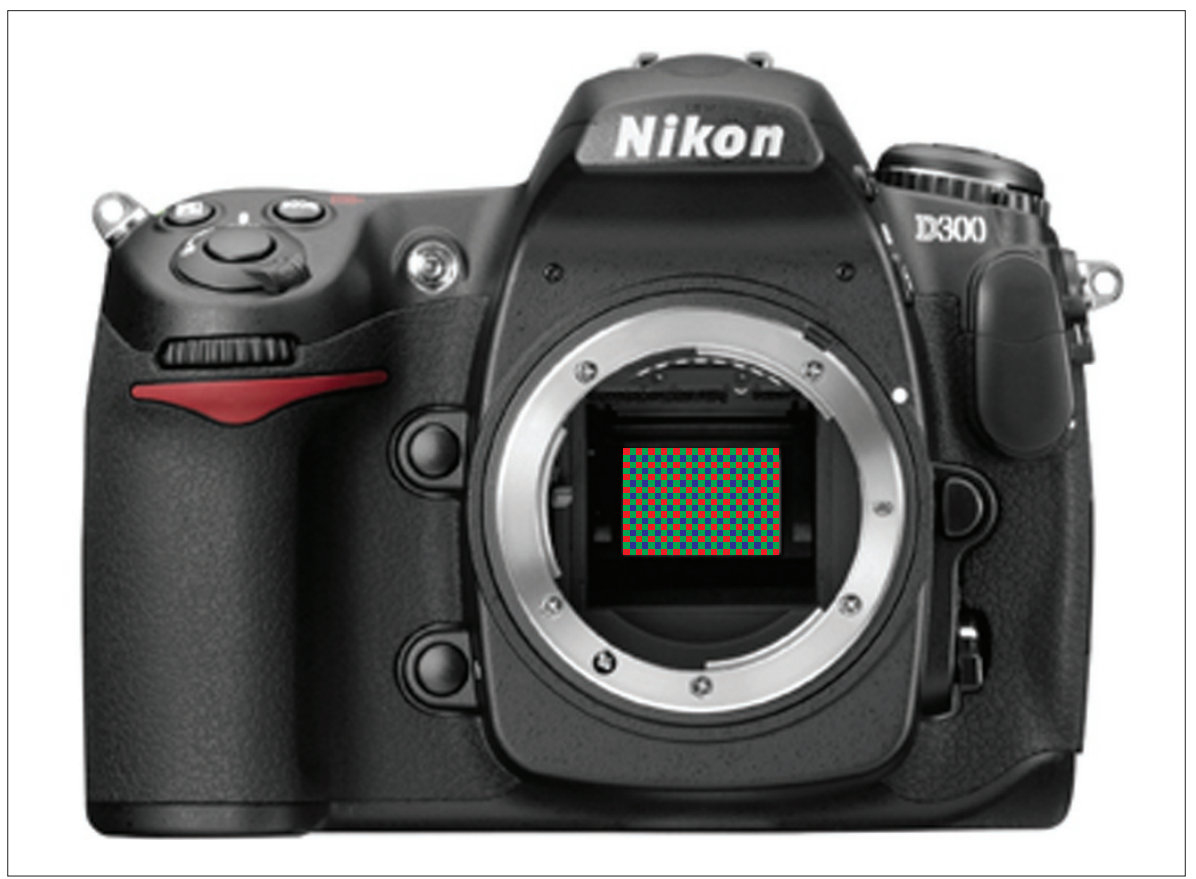

Fig. 8 The body of a DSLR houses the CCD sensor and electronics

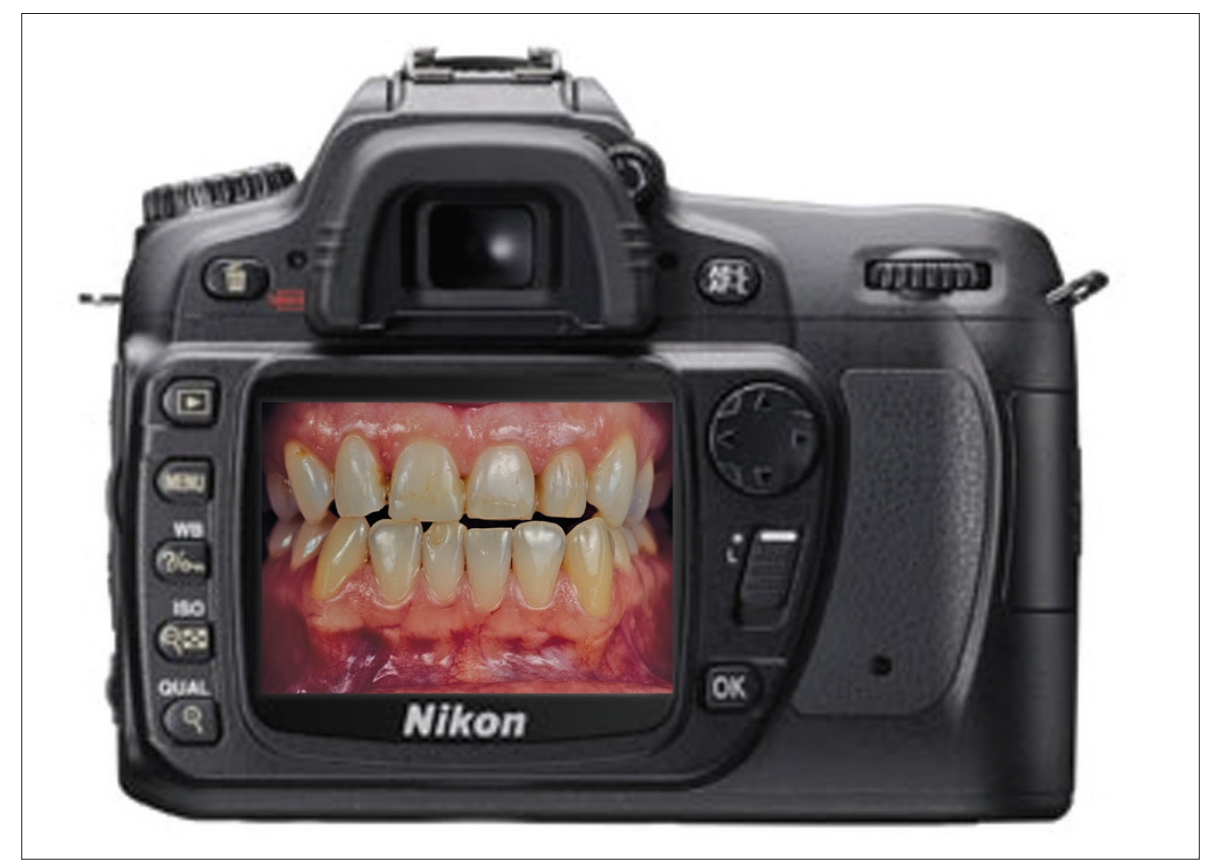

Fig. 9 The LCD viewer on the camera back allows a video display of the intended image, followed by instant viewing of the image after exposure 


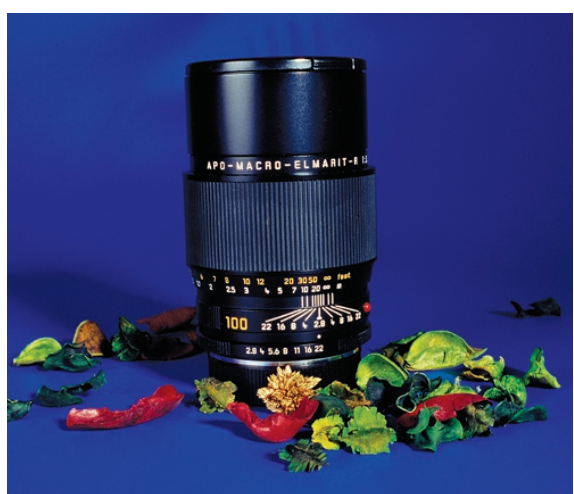

Fig. 10 A high quality macro-telephoto is the ideal lens for dental photography

teeth or parts of individual teeth may be missing on the resultant image. Some manufacturers have ingeniously overcome parallax by viewing and focusing attachments, all with limited success. This is analogous to buying a family saloon car and then customising it for formula one racing, which seems a little futile!

\section{DIGITAL SINGLE LENS REFLEX}

The most versatile camera for dental photography and for achieving the best results is without doubt a DSLR system. A DSLR offers TTL (through the lens) viewing and metering, precise focusing and accurate framing. The major advantage of DSLRs is that parallax is eliminated, because the viewfinder, lens and image sensor all share the same optical axis. This means that what is seen in the viewfinder is identical to that recorded on the resulting image.

A DSLR consists of a camera body housing the sensor, LCD viewer and microprocessor, which is the 'brain' of the camera (Figs 8-9). At one time it was a luxury to have a live video display on the LCD, but nowadays this feature is almost commonplace. However, it should be remembered that the video display lags behind what is observed in the viewfinder depending on the refresh rate of the LCD screen. The second component is the interchangeable lenses, which are selected according to the type of photographic application, for example macro, portrait, landscape, sports, wildlife, etc. A few DSLRs have fixed zoom lenses, which are usually incapable of taking macro dental images. The standard lens of a DSLR has a focal length of $50 \mathrm{~mm}$; a shorter focal length lens, say $28 \mathrm{~mm}$, is classified as wide angled (for example, for landscapes), while a longer focal length is a telephoto (for example, for sports or wildlife).

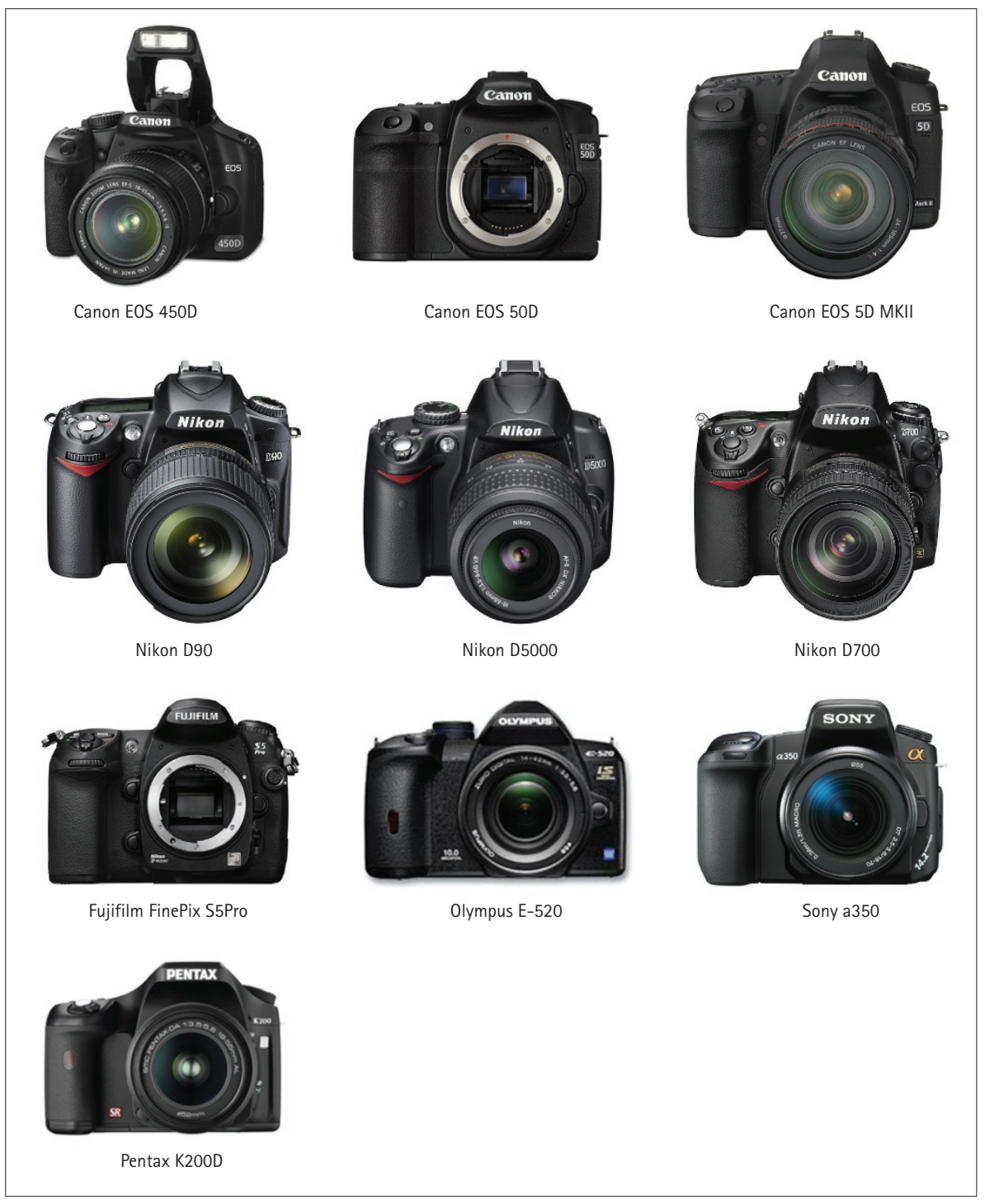

Fig. 11 A selection of semi-professional DSLR cameras, all approximately $€ 500$

For dental applications, a dual-purpose lens is necessary, firstly for portraiture and secondly to focus down for close-up photography. The ideal choice is therefore a lens that combines both these features, that is, a macro-telephoto lens. A word of caution about macro lenses. Many lenses have 'macro' etched on their barrels but are not true macro lenses. Even compact cameras claim macro facilities but this only indicates close focusing facilities. A true macro is capable of producing a 1:2 or 1:1 magnification. A 1:1 magnification is the ideal and means that the image recorded by the sensor is the same size as the object in real life. For $35 \mathrm{~mm}$ format DSLRs, a 1:1 image usually translates to about four maxillary incisors.

Depending on the manufacturer and arrangement of optics within the lens barrel, the focal lengths of macro-telephotos vary from $50 \mathrm{~mm}$ to $105 \mathrm{~mm}$. Also, many sensors are smaller than the $35 \mathrm{~mm}$ film format, and therefore have a multiplication factor. For example, attaching a $100 \mathrm{~mm}$ lens to a $35 \mathrm{~mm}$ camera body will effectively 'convert' the focal length of the lens to $150 \mathrm{~mm}$, that is, the sensor has a multiplication factor of 1.5 . However, some newer high-end cameras have larger sensors and therefore the lenses do not require a multiplication factor. It is important to purchase the highest quality lens that you can, preferably a proprietary make rather than a third party analogue. The lens characteristics (resolution and superior optics) are essential for high quality images, and spending a little extra money in the beginning will pay dividends in the long term. The lens characteristics should include high contrast for higher resolution, combined with superior optical quality for minimising chromatic and spherical distortions. Lenses with the prefix 'APO' (Fig. 10) and 'ASPH' indicate 
low chromatic and spherical aberrations, respectively. Finally, auto-focus lenses may not operate in some close-up dental set-ups, and the ability to revert to manual focusing is a useful option.

Most semi-professional DSLRs offer all the specifications necessary for dental photography (Fig. 11). The salient features to scrutinise before purchasing a camera are:

1. Image sensor: CCD or CMOS

with greater than six megapixels.

However, most current semi-

professional cameras exceed ten megapixels as standard (see Part 3)

2. Bit depth: minimum 8 bit/channel (24 total bit or colour depth), 16 bit/ channel (48 total bit or colour depth) preferred (see Part 3)

3. Dynamic range of sensor: minimum $6 \mathrm{f}$ stops, more than $6 \mathrm{f}$ stops preferred (covered further in Part 6)

4. Dust reduction system for sensor: mitigating unwanted particle build-up on sensor or access to the sensor for cleaning

5. Metering: multi-pattern TTL with aperture priority mode (to be covered in Part 6)

6. Flash metering: TTL synchronisation (to be covered in Part 5)

7. White balance: automatic and manual (to be covered in Part 6)

8. Full frame capture (not mandatory if cost is prohibitive)

9. ISO range: ability to set a minimum of 100 for low noise (to be covered in Part 6)

10. Data formats: RAW, PNG, TIFF and JPEG files (to be covered in Part 9)

11. Colour domains: Adobe RGB and sRGB (to be covered in Part 6)

12. Storage media: maximum internal capacity or memory cards, greater than one gigabyte (see Part 2)

13. Interface: FireWire or other high speed transfer to computer (to be covered in Part 9).

It is difficult to recommend manufacturers or models of cameras, since the market is rapidly changing and new products are introduced annually or even every few months. The best way to choose a camera is to visit a retail showroom and check that a semi-professional DSLR meets the above criteria and specifications. Of course one can purchase high-end professional DSLRs

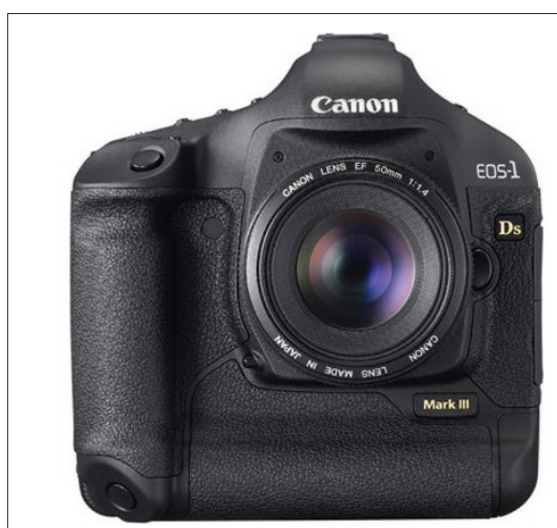

Canon EOS 1Ds MKIII

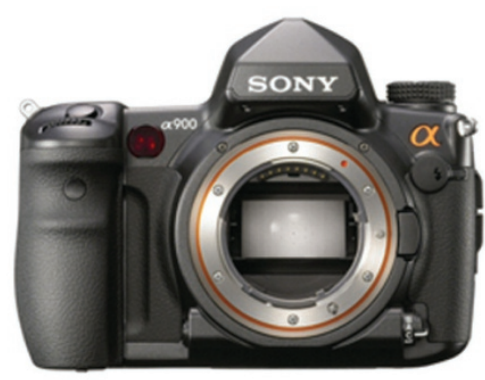

Sony a900

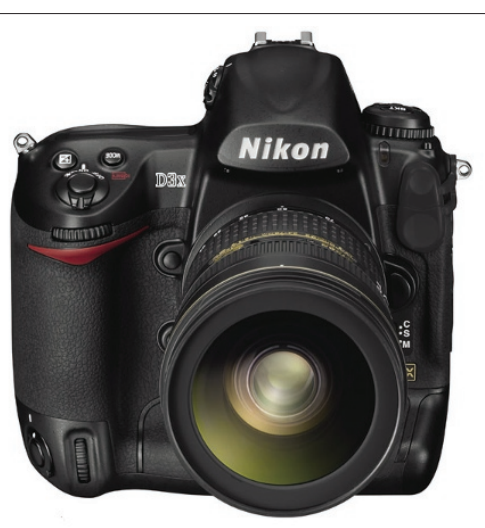

Nikon DX3

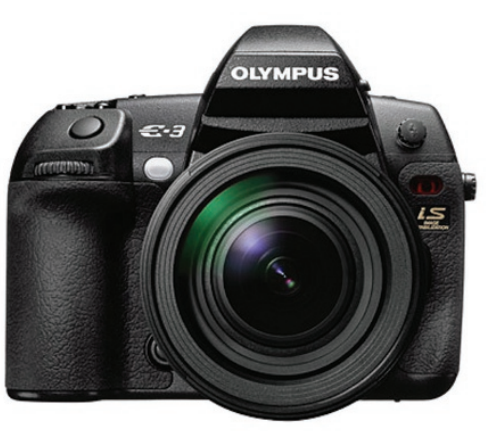

Olympus E-3

Fig. 12 A selection of professional DSLR cameras, from $€ 800$ to $£ 6,000$

(Fig. 12) with additional features, some gimmickry and functions that are superfluous for dentistry. Unless one is fastidious about image quality, it is pointless spending ten times the amount purchasing equipment with features that one will rarely use. However, to ensure a prudent long-term investment, when choosing a lens it is preferable to opt for a proprietary macro-telephoto lens of the highest optical quality. This ensures that even if the camera body becomes obsolete, the lens can still be used with a newer body purchased in the future.

\section{IMAGE QUALITY}

A key factor before choosing photographic equipment is ensuring that the items purchased harmoniously integrate and 'communicate' with each other to yield acceptable image quality. The theoretical aspect of image quality was discussed in Part 3, but now requires further elaboration in relation to the photographic hardware. Image quality is influenced by perceptual and practical factors.

\section{Perceptual factors}

Image quality is a subjective and nebulous entity meaning different things to different people. Summarised succinctly, image quality depends on the following items, which are briefly discussed below:

1. Magnification

2. Inherent object details

3. Degree of the trained eye

4. Visual acuity and acutance

5. Psychological perception of detail

6. Circle of confusion

7. Distance of viewing

8. Viewing media.

Magnification and inherent object details (dependant of illumination) are discussed in Part 3 and Part 5, respectively.

An individual trained to 'see' certain details will perceive them more than an untrained layman. This is clearly evident in dental aesthetic assessment. A clinician's eye is trained to scrutinise details pertaining to dental aesthetics far more than the average patient. Visual acuity or perception of sharpness is a function of the eyes, which deteriorates with advancing years and is compensated by eyeglasses or magnification aids. A physical measurement of sharpness is performed by densitometers and is termed acutance. The latter is an objective 
measure of sharpness, not influenced by subjective idiosyncrasies.

Psychological perception of detail varies enormously, not only from individual to individual, but also intra-individually. An individual will see what his or her brain wants to perceive, depending on their social, academic, religious and intellectual psychological make-up. Furthermore, an individual may perceive the same image differently at different times depending on his or her state of mind. Factors such as stress, lethargy, influence of intoxicating substances (alcohol, psychotropic drugs), insomnia, inactivity and libido changes all affect an individual's ability to discern detail.

The circle of confusion is a phenomenon that also affects image quality. Any lens, no matter how perfect, will always represent a perfect pinpoint as a tiny blur. The circle of confusion can be reduced, but never to the original pinpoint. Therefore, an acceptable dimension is assigned to represent the original pinpoint, which varies from $0.1 \mathrm{~mm}$ to $0.033 \mathrm{~mm}$, depending on different authorities and manufacturers. The significance of the circle of confusion is that the nearer the viewer is to an image, the smaller the circle of confusion necessary to ensure image sharpness. For example, viewing an A4 image requires a smaller circle of confusion compared to viewing a $20 \times 10$ foot billboard poster from a distance. The same is true for watching a film in a cinema: the further away from the screen, the sharper the image.

Additionally, viewing media also alter image detail. For example, a printed image which is converted from an RGB original to CMYK separations will have obvious detail loss compared to the original image.

Finally, appearance is also affected by the five modalities of colour:

1. Object colour - colour due to white light reflected off its surface, for example a tomato appears red because all colours of the spectrum are absorbed except the colour red, which is reflected off its surface

2. Volume colour - objects such as coloured wine bottles, where the colour is related to the volume of the object

3. Aperture colour - colour of a space, for example blue sky

4. Illumination colour - transmission of

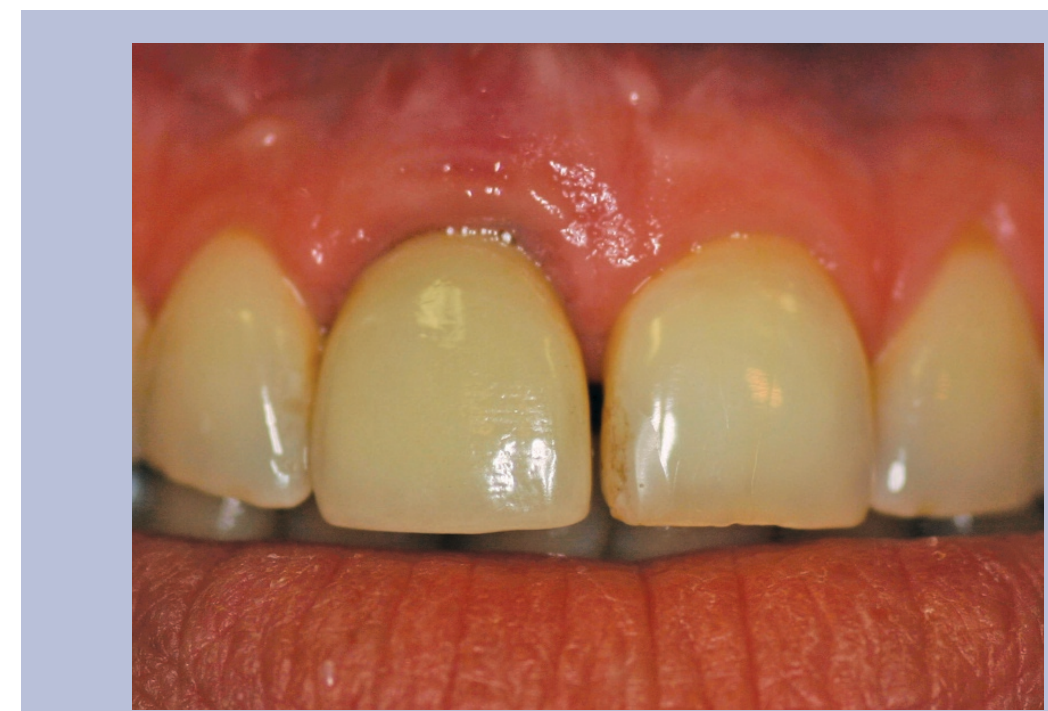

Poor image quality with inexpensive 10 megapixel camera, there is little detail of the soft tissues texture and poor depiction of tooth characterization

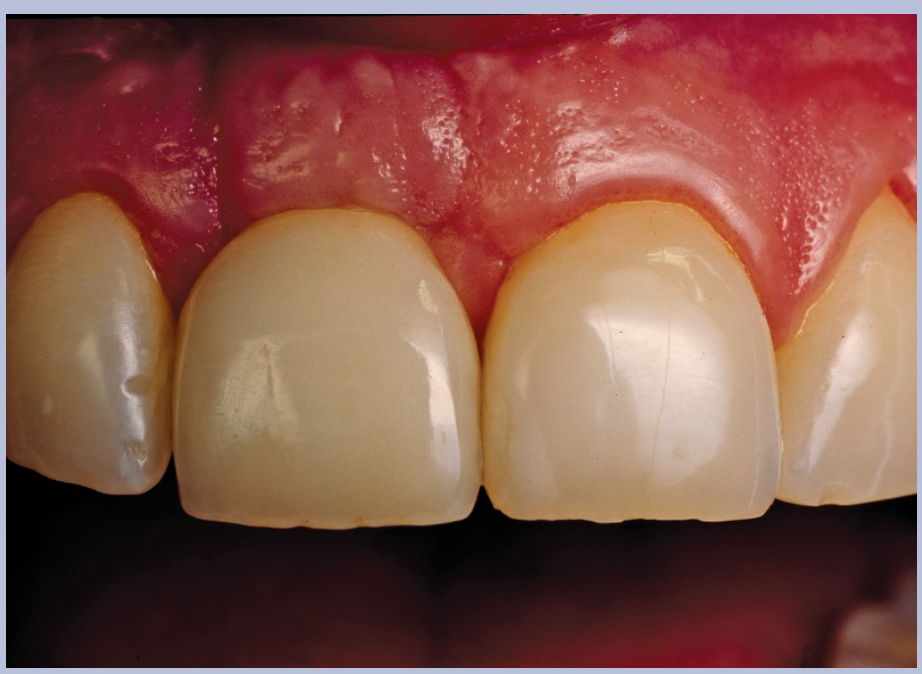

High image quality with expensive 10 megapixel camera, the soft tissue architecture and gingival stippling is clearly visible, as well as tooth stains, and fracture lines

Fig. 13 The number of pixels alone does not influence image quality. Both images are taken with 10 megapixel cameras. The top image, using an inexpensive camera, has little detail and a low dynamic range. The bottom image is with an expensive camera, and is rich in detail with a high dynamic range

white light through a coloured surface, for example viewing transparencies using a projector

5. Illuminant colour - an incandescent entity, such as a light bulb, emanating light of a specific hue.

\section{Practical factors}

The single feature about digital cameras most frequently quoted by manufacturers and retailers is the number of megapixels. But contrary to popular belief, the numbers of pixels does not determine the quality of a digital image: they determine the size of an image.

The hardware, and subsequent software processing and manipulation determine image quality. Returning to the acronym CPD (capture, processing, display) discussed in Part 3, how good the image looks is determined by method of capture (a function of lens quality together with the quality and quantity of pixels), method of in-camera processing (A/D converter) and the method used to display an image (monitor, prints). 


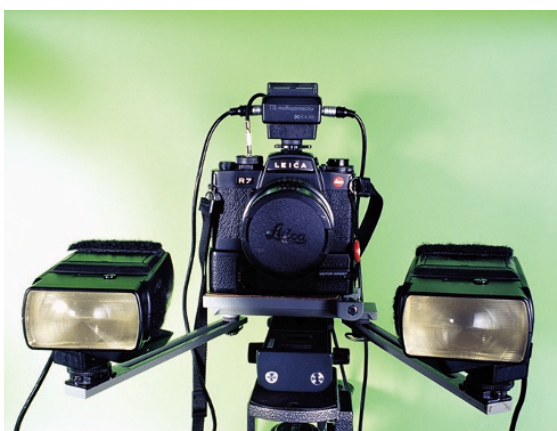

Fig. 14 The tripod supports a mechanical stage, flash brackets and the camera. A TTL adaptor mounted on top of the camera controls the bilateral flashes and exposure is made with cable release

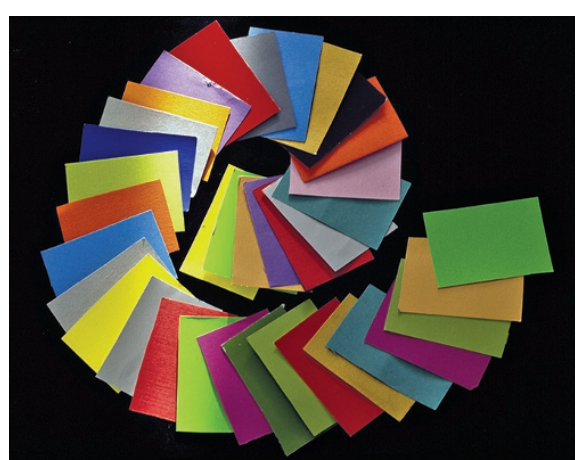

Fig. 15 A selection of coloured cards used as backdrops for photographic set-ups in a dental laboratory

\section{Capture}

The predominant factor affecting image quality is the optics of the lens, discussed previously. Furthermore, as the physical pixel size decreases to cram more onto the limited surface area of a sensor, the demands on the lens to resolve detail increases. For example, purchasing a digital camera with a 10 megapixel sensor and attaching a lens capable of only resolving 6 megapixels of detail will do little to increase image quality. The quality of the pixels is determined by the type of sensor, either CCD (charge coupled device) or CMOS (complementary metal oxide semi-conductor). CCD sensors allow a larger bit depth (range of colours), higher dynamic range (increased contrast) and greater signal to noise ratios (avoiding grainy images). The CMOS are secondgeneration sensors with less power consumption, which allow access to individual pixels. Pictures taken with two different cameras with the same number of pixels will vary enormously because the quality of pixels is a crucial factor determining image quality. It is possible to purchase a relatively inexpensive compact camera

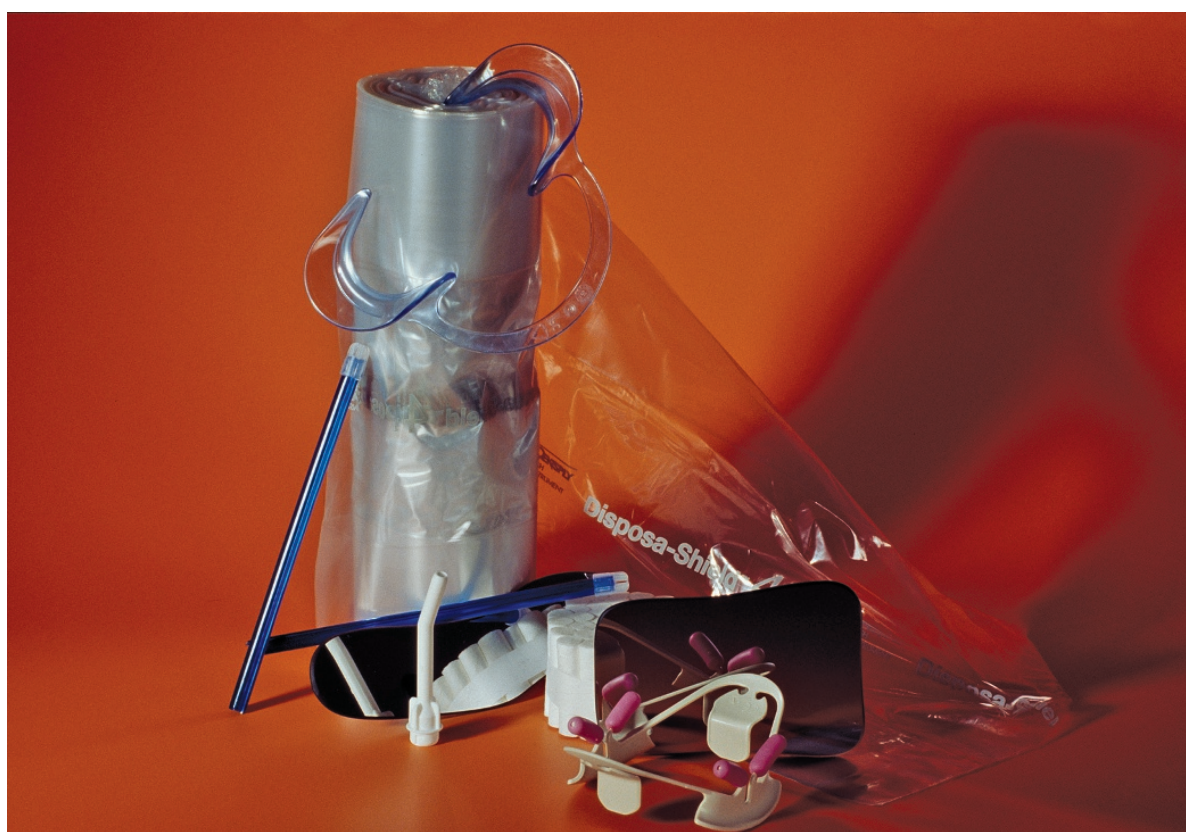

Fig. 16 The dental armamentarium required for dental photography

with 10 megapixels and one which is ten or twenty times the price, also with 10 megapixels. Both cameras will capture an image that is identical in size. However, the image from the inexpensive camera will be poorer in quality compared with that from the expensive camera. Although both cameras have the same number of pixels, to ensure high image quality other factors much be taken into account such the resolving power of the lens, the A/D converter, pixel quality, pixel size, bit depth, dynamic range, file format, degree of noise and method of display (Fig. 13).

To summarise, the number of pixels determines the size, not quality, of an image. However, the numbers of pixels become significant when enlarging an image. For example, for a print size of $5 \times 7$ inches, a camera with a 3 megapixel sensor is adequate. If larger images are required, or enlargement of a part of the image is necessary, more pixels are necessary to avoid deterioration of the image quality. For dental photography, 6 megapixels are more than sufficient, allowing a high quality A4 print assuming that the image is printed without magnification.

\section{Processing}

The only method of recording a virgin, or pure analogue image signal is to record it on film. All software and hardware used for processing, to a lesser or greater degree adulterate the captured image signal. In most contemporary digital cameras, the corruption is insignificant and rarely perceptible. However, the in-camera software, which eventually extrapolates a picture from the image capture, should be sufficiently sophisticated to minimise flaws. For example, if the initial capture is 24-bit, but is processed with a 12-bit depth A/D converter, 12 bits of information or detail are lost. Furthermore, if in-camera processing is set to translate a RAW image to a low quality JPEG file format, further loss of information is inevitable.

\section{Display}

Finally, the method of display has a bearing on how the final image is perceived. Once again, image quality is affected by the resolving power of the monitor (CRT or LCD), the type of file used to display the image, the quality of paper and the printing equipment, or the calibration [and intensity] of a projector. For the untrained eye and for most dental applications, a computer monitor, inkjet printer with photographic paper or standard 'beamer' projectors are acceptable. It is only the aficionados who will detect nuances which necessitate calibration of monitors, projectors and printers to optimise the final display.

\section{PHOTOGRAPHIC ACCESSORIES}

Besides the camera and lens, other photographic adjuncts are useful accessories to expedite dental photography. 
Camera supports allow hands-free, no touch protocols to be practised. This not only offers convenience, but is also conducive to sterility and disinfection procedures in the clinical environment. A tripod is an essential support item to stabilise the camera, and other mounted accessories and various types are available offering sturdiness on a solid platform.

A tripod ensures precise picture framing and focusing and is conveniently moved aside once a photo session is finished. Besides tripods, if space is a paramount concern there are numerous ingenious camera support devices on the market, which can be tailored to specific surgery requirements. A visit to the local camera shop or surfing the Internet provides ample ideas and choices.

As well as securing the camera to a tripod, flashes can be mounted either laterally or superiorly to the camera body via brackets, for example the macro flash bracket (Manfrotto, Italy). Finally, the camera and flash bracket can be supported on the tripod via a graduated mechanical stage (Kaiser Phototechnik, Germany) as in Figure 14. A stage also facilitates precise focusing, especially when a particular scale of reproduction is required, for example 1:1 or 1:2. Additionally, no contact with the lenses is necessary, which also benefits a 'no touch' protocol. This set-up is also useful for extra-oral bench photographs of dental casts in the dental laboratory. For photographing radiographs a copy stand is indispensable, ensuring precise location and uniform illumination (Kaiser Phototechnik, Germany).

Another accessory is a remote release cable, which can be wireless or foot controlled and is invaluable for taking pictures of surgical procedures where cross-infection control is essential. Also, an 18\% grey card and a photographic colour guide is useful for dental shade analysis and white balance calibration, discussed further in Part 6.

The purpose of backdrops, or backgrounds, is to isolate and concentrate attention on the object being photographed. The oral environment serves as a natural backdrop and due to limitations of space and access, in vivo backgrounds are rarely required. On occasions, a black card placed behind the maxillary anterior teeth may be useful for highlighting translucency and incisal edge characterisations.

For portraiture and extra-oral pictures, backgrounds are a requisite and add interest to the composition. However, subtlety is necessary when choosing backgrounds, since flamboyant, ostentatious or lurid backgrounds detract attention from the main subject. Any medium such as cloth, coloured papers, walls or furniture can effectively be utilised to separate and therefore highlight the main object. A background consisting of a cluster of objects causes visual confrontation and is distracting, for example photographing a patient seated in a dental chair with the entire surgery armamentarium serving as a backdrop.

For dental laboratory or bench photography, backgrounds are extremely useful for blocking extraneous objects or the laboratory equipment and clutter. The simplest backdrops are coloured cards, cut to various sizes, illuminated separately from the main subject, giving a sense of separation and three dimensionality (Fig. 15). Another useful medium is cloth such as black velvet, which absorbs all incident light, producing a uniform black background. Textured or patterned backgrounds should be avoided unless a special effect is required. Professional still life shooting tables and props with custom-made backgrounds are commercially available for expediting repetitious set-ups.

\section{DENTAL ARMAMENTARIUM}

The main items needed for intra-oral photography are cheek retractors, available from dental suppliers in unilateral and bilateral varieties. Traditionally, metal retractors were universally used for dental photography, but they are more traumatic than the plastic pliable varieties that are generally preferred. The bilateral variety is used for talking pictures of anterior teeth, while the unilateral type is useful for lateral views and pictures of posterior teeth. Intra-oral photographic mirrors are necessary for occlusal, lingual (or palatal) and lateral views of teeth. Dental photographic mirrors should be front-coated to avoid double images. Several sizes are available to accommodate various degrees of mouth opening.

Other dental items necessary for photography are readily available in a dental surgery. These include cotton wool rolls, saliva ejectors and rubber dam for isolation and moisture control (Fig. 16). An oil-free, 3-in-1, or 6-in-1 syringe delivering warm air ensures that the soft tissues and teeth are free of saliva and blood and prevents condensation or fogging onto the surface of intra-oral mirrors. Another approach to preventing condensation on mirror surfaces is to pre-soak in warm water before use. Plaque and food particles are removed by flossing and polishing with prophylaxis paste before a photographic session (unless the intention is recording biofilm or extrinsic stains). Gingival bleeding or crevicular exude, for example following crown preparation, is arrested with retraction cord soaked in a haemostatic agent, for example buffered aluminium chloride. Astringent agents containing adrenaline or ferric compound are avoided to prevent cardiac stimulation and black gingival residue, respectively. 